\title{
Binary pathways to SLSNe-I: SN 2017gci
}

\author{
H. F. Stevance, ${ }^{1 \star}$, J. J. Eldridge ${ }^{1}$ \\ ${ }^{1}$ The Department of Physics, The University of Auckland, Private Bag 92019, Auckland, New Zealand
}

Accepted XXX. Received YYY; in original form ZZZ

\begin{abstract}
Some hydrogen poor superluminous supernovae (SLSNe) exhibit bumps in the tails of their light-curves associated with hydrogen features in their late time spectra. Here we use the explosion parameters of one such SLSN - SN 2017gci - to search the stellar models of the Binary Population And Spectral Synthesis (BPASS) code for potential progenitors. We find good matches for a $30 \mathrm{M}_{\odot}$ progenitor star in a binary system and no matches from single star models. Common envelope and mass transfer after the giant branch, combined with increased mass loss from strong stellar winds immediately before death, allow the progenitor to lose its hydrogen envelope decades before the explosion. This results in a hydrogen poor SLSN and allows for delayed interaction of the ejecta with the lost stellar material.
\end{abstract}

Key words: transients: supernovae - supernovae:SN2017gci - stars:evolution

\section{INTRODUCTION}

Superluminous supernovae (SLSNe) are a class of astronomical transients separated from standard supernovae by a luminosity threshold of $\mathrm{M}_{g}<-19.8 \mathrm{mag}$ (Gal-Yam 2019). Similarly to their less luminous counterparts, SLSNe come in two main flavours: hydrogen rich (SLSNe-II) and hydrogen poor (SLSNe-I). The excess brightness of type II SLSNe is likely powered by interaction of the ejecta with hydrogen rich circumstellar material (CSM - see Gal-Yam 2019 for more details), whereas the case of the SLSNe-I still remains under debate. The most commonly invoked mechanism is the magnetar model in which the light curve is powered by the spin-down of a strongly magnetic $\left(\mathrm{B} \sim 10^{14-15} \mathrm{G}\right)$, rapidly rotating $(\mathrm{P} \sim 1-4 \mathrm{~ms})$ central engine (Woosley 2010; Kasen \& Bildsten 2010; Nicholl et al. 2017). This model has been largely successful at reproducing the early lightcurves of hydrogen poor SLSNe, particularly those with smooth decay tails (e.g. Dessart et al. 2012; Inserra et al. 2013; Nicholl et al. 2015).

There is, however, a number of hydrogen poor SLSNe which show oscillations in their late time lightcurve (a few tens to over 100 days after maximum, e.g iPTF13ehe, SN2015bn, iPTF15esb, iPTF16bad, SN2017gci- Yan et al. 2015; Nicholl et al. 2016; Yan et al. 2017; Fiore et al. 2021), some of which accompanied by detections of $\mathrm{H} \alpha$ in their spectra. The presence of these features could be a sign of interaction with CSM (Yan et al. 2017; Inserra et al. 2017), e.g. from pulsational pair instability a few decades before explosion (iPTF13ehe, Yan et al. 2015 - iPTF16ehe, Lunnan et al. 2018). Alternatively Moriya et al. (2015) developed a binary model whereby the broad hydrogen features $\left(\sim 4500 \mathrm{~km} \mathrm{~s}^{-1}\right.$ in Yan et al. 2015$)$ are the result of mass stripping from a binary companion by the supernova ejecta. The hydrogen material is initially hidden within the photosphere, but as the opacity decreases, the hydrogen is revealed during the nebular phase.

\footnotetext{
$\star$ E-mail: hfstevance@gmail.com
}

In this work we focus on the recently published SN 2017gci (Fiore et al. 2021), which showed two rebrightenings at $\sim 103$ and $\sim 142$ days after maximum light and the emergence of $\mathrm{H} \alpha$ from +51 days to $\sim 133$ days. Fiore et al. (2021) performed fits on the photometric data using three models: a nickel powered, magnetar powered and CSM powered lightcurve. The nickel model was excluded as it required a larger nickel mass than the derived ejecta mass for SN 2017gci; a CSM model with $M_{\mathrm{ej}} \approx 12 \mathrm{M}_{\odot}$ and $M_{\mathrm{csm}} \approx 5 \mathrm{M}_{\odot}$ can reproduce the peak, but would require an additional power source to explain the tail; a magnetar model with initial period $\mathrm{P} \approx 2.8 \mathrm{~ms}$, and ejecta mass $M_{\mathrm{ej}} \approx 9-10 \mathrm{M}_{\odot}$, on the other hand, provided a good fit to the peak and the tail. We seek to find models that can give rise to the progenitor and environment of SN $2017 \mathrm{gci}$. Here we focus on the scenario where the bumps and associated hydrogen features originate from CSM around the progenitor (Fiore et al. 2021).

We use the reported parameters of the explosion to create a set of criteria and search existing stellar models in the Binary Population and Spectral Synthesis (BPASS - Eldridge et al. 2008, 2017; Stanway \& Eldridge 2018) code. These simulations have a wide scope and have been used in a large array of applications: to better understand and age stellar populations (e.g. Wofford et al. 2016; Stevance et al. 2020b; Brennan et al. 2021), study nebular emission (e.g Xiao et al. 2018, 2019), investigate the reionization of the universe (e.g Stanway et al. 2016; Ma et al. 2016), and predict binary black-hole mergers (Eldridge \& Stanway 2016), to name a few. Our main goal is to check whether matching progenitors can be found natively in stellar evolution models that self-consistently recreate the characteristics of stellar populations found in the Universe.

In Section 2 we give a brief description of the data used in this work, in Section 3 we lay out a set of search criteria based on the explosion parameters of SN 2017gci and the literature. In Section 4 we describe the matching models, these results are discussed in Section 5 before concluding and summarising our findings in Section 6 . 
Table 1. Summary of the model search constraints and the number of stellar models left after each is applied. In the third column the number of models are given for an explosion energy $10^{52} \mathrm{ergs}\left(10^{51} \mathrm{ergs}\right)$.

\begin{tabular}{lll}
\hline Property & Constraint & Number of models \\
\hline $\mathrm{Z}$ & 0.006 & 21,598 \\
$\mathrm{M}_{\text {ZAMS }}$ & $>10 \mathrm{M}_{\odot}$ & 9476 \\
$\mathrm{M}_{\text {rem }}$ & $<2.43 \mathrm{M}_{\odot}$ & $9233(5155)$ \\
$\mathrm{M}_{\mathrm{ej}}$ & $8-13 \mathrm{M}_{\odot}$ & $1863(774)$ \\
$\mathrm{X}$ (at death) & $<0.01$ & - \\
$\mathrm{M}_{\boldsymbol{H}}$ (at death) & $<0.001 \mathrm{M}_{\odot}$ & $761(2)$ \\
$\mathrm{t}_{\mathrm{csm}}$ & $<150$ years before death & 2 (None) \\
\hline
\end{tabular}

\section{MODEL DATA}

We use the stellar models of BPASSv2.2.1 ${ }^{1}$ which are computed for 13 metallicities and 9 different initial mass functions (IMF - for more details see Eldridge et al. 2017; Stanway \& Eldridge 2018). Here we only consider the fiducial IMF: a Kroupa prescription with maximum mass $300 \mathrm{M}_{\odot}$ (Kroupa 2001). BPASS creates a population of stars amounting to $10^{6} \mathrm{M}_{\odot}$ according to the chosen IMF and evolves them over 51 time bins ranging form $\log$ (age/years) $=6.0$ to $\log$ (age/years $)=11.0$ in increments of $0.1 \mathrm{dex}$. This includes singlestar-only models as well as binary models; here we focus on the latter. It is important to note that these binary models also contain single stars (and effectively single stars) that do not undergo binary interaction, as is expected in the Universe. Additionally, the distribution of initial periods and mass ratios are based on observations (Moe \& Di Stefano 2017). In the following searches we will not explicitly select for binary or single system.

BPASS currently includes $\sim 250,000$ stellar models, each recorded in one text file compiling over a hundred properties over dozens to hundreds of time steps. With modern machines, computational bottlenecks often reside in memory and disk access rather than the number of floating point operations that processors can handle. In order to avoid repeatedly opening and reading thousands of text files we used hoki (Stevance et al. 2020a) to compile the stellar models into a data product better suited to data analysis within python. Specifically the data were split into 26 binary files each containing one pandas dataframe (pandas development team 2020; Wes McKinney 2010) comprised of all the stellar models for one metallicity and one simulation type - either single star models only, or including binaries (Stevance 2020) ${ }^{2}$. These data products can then be searched quickly and efficiently using existing python infrastructure and they are made widely accessible so that similar analyses can be performed by others in the future.

\section{SEARCH CRITERIA}

\subsection{Basic constraints}

First we restrain our search to a single metallicity since Fiore et al. (2021) estimate that the host galaxy of SN 2017gci has Z $\sim 0.3 \mathrm{Z}_{\odot}$ based on strong line indicators (D02, PP04 O3O2, PP04 N2Ha,M13N2 Denicoló et al. 2002; Pettini \& Pagel 2004; Maiolino et al. 2008; Marino et al. 2013). Assuming a solar metallicity mass fraction of 0.02, the closest match in BPASS are the $\mathrm{Z}=0.006$ models.

Next, we apply a MZAMS mass cut-off. Fiore et al. (2021) state

\footnotetext{
1 https://drive.google.com/drive/folders/

1BS2w9hpdaJeul6-YtZum--F4gxWIPYXl

2 https://zenodo.org/record/4064300\#.YDyH5nVfgYt
}

that the progenitor is likely over $40 \mathrm{M}_{\odot}$ but this is based on the single star models of Jerkstrand et al. (2017), and so as not to bias this search a lower mass constraint of $10 \mathrm{M}_{\odot}$ is chosen. In theory no mass cut-off need to be applied, but in practice some of the following steps are unnecessarily computationally intensive without this limit. This makes the data more manageable without introducing a bias since the ejecta mass in Fiore et al. (2021) is $\sim 10 \mathrm{M}_{\odot}$ and our limit is therefore very conservative.

\subsection{Ejecta and remnant mass}

Next the ejecta mass and the remnant mass are constrained. The former is narrowed down by Fiore et al. (2021) to $M_{\mathrm{ej}} \approx 9 \mathrm{M}_{\odot}$ or $M_{\mathrm{ej}} \approx 12 \mathrm{M}_{\odot}$ for the magnetar and CSM models respectively. The former is preferred as it can explain the peak and tail of the lightcurve of SN 2017gci. Not knowing the full extent of the uncertainties on these ejecta masses we keep our search wide and constrain our $M_{\text {ej }}$ to be between $8 \mathrm{M}_{\odot}$ and $13 \mathrm{M}_{\odot}$.

Since a magnetar is also required, the remnant mass is limited by the maximum mass of a neutron star (NS). This upper limit remains uncertain but studies based on the analysis of the NS-NS merger GW170817 (Abbott et al. 2017, 2019) report a 90 percent upper bound at $2.43 \mathrm{M}_{\odot}$ (Abbott et al. 2018); other works by e.g. Essick et al. (2020) find a slightly lower mass, around $2.3 \mathrm{M}_{\odot}$ (for an extensive discussion of the uncertainty on the NS mass limit see section 6.2 of Abbott et al. 2020). For the purposes of this search $2.43 \mathrm{M}_{\odot}$ was used as the limiting value, but given the remnant masses of the model matches $\left(\sim 1.4 \mathrm{M}_{\odot}\right.$, see Section 4$)$, the uncertainty in the NS maximum mass is not a cause of concern.

It is important to remark that the remnant mass and ejecta mass in BPASS (and in the Universe) are dependent on the energy output of the explosion. In our models there are three sets of ejecta and remnants masses corresponding to "weak" ( $\left.10^{50} \mathrm{ergs}\right)$, "normal" $\left(10^{51} \mathrm{ergs}\right)$ and "super" (10 52 ergs) supernovae explosions - note these keywords are used in the BPASS manual and in the hoki data products. Nicholl et al. (2017), for a sample of 38 hdyrogen poor SLSNe, found kinetic energies ranging from $2 \times 10^{51}$ to $10^{52}$, so in the rest of this study we ignore the "weak" explosions.

\subsection{Hydrogen content at death}

Because SN 2017gci is a SLSN-I, the hydrogen levels of its progenitor at death must be close to none. Specifically, simulations by Dessart et al. (2012) showed that strong $\mathrm{H} \alpha$ lines can be present in supernovae spectra with a hydrogen mass fraction $(\mathrm{X})$ of 0.01 and a total hydrogen mass $\left(\mathrm{M}_{H}\right)$ of $0.001 \mathrm{M}_{\odot}$. These values are used here as boundaries between hydrogen "rich" and hydrogen "poor" progenitors.

\subsection{Hydrogen content before death}

The presence of bumps in the lightcurve of SN 2017gci as well as $\mathrm{H} \alpha$ signatures in its spectra between phase 51 and 133 days are interpreted as the presence of hydrogen rich CSM, potentially in shells (Fiore et al. 2021). If this CSM originates from the progenitor of SN $2017 \mathrm{gci}$, we can roughly estimate when the final layers of hydrogen must have been lost by following

$\mathrm{t}_{\mathrm{csm}}=\frac{\mathrm{v}_{\text {phot }} \times \mathrm{t}_{\text {interact }}}{\mathrm{v}_{\mathrm{csm}}}$,

where $t_{c s m}$ is how long before the explosion the CSM was ejected, $\mathrm{v}_{\text {phot }}$ is the photospheric velocity (estimated by Fiore et al. (2021) to 
be $\sim 8000 \mathrm{~km} \mathrm{~s}^{-1}$ ), $\mathrm{t}_{\text {interact }}$ is the time with respect to explosion when the ejecta encounters the CSM, and $v_{\mathrm{csm}}$ is the velocity of the CSM.

Not all of these quantities are known, specifically the CSM velocity is not quantified in Fiore et al. (2021), and the explosion date is uncertain. Based on SLSN-I samples from the PAN-STARRS1 Survey and the Palomar Transient Factory, the rise time of this type of explosion ranges from as low as 15 days to $\sim 100$ days (De Cia et al. 2018; Lunnan et al. 2018). In the case of SN 2017gci, the rising phase of the lightcurve is not captured; for our estimates we focus on two cases, $\mathrm{t}_{\text {rise }} \sim 50$ days and $\mathrm{t}_{\text {rise }} \sim 100$ days, and since the first sign of $\mathrm{H} \alpha$ is seen at 51 days after maximum, these correspond to $t_{\text {interact }}$ of 100 and 150 days. Finally, for the CSM velocity, a slow and a fast wind scenario are considered: $\mathrm{v}_{\mathrm{csm}}=100 \mathrm{~km} \mathrm{~s}^{-1}$ and $\mathrm{v}_{\mathrm{csm}}=1000$ $\mathrm{km} \mathrm{s}^{-1}$. Wind velocities of heavily stripped WR stars can easily surpass the velocity of the fast wind case (e.g. $5000 \mathrm{~km} \mathrm{~s}^{-1}$ - see Tramper et al. 2015 and references therein), but here we are interested in an order-of-magnitude estimate.

For $t_{\text {interact }}=100$ days the final layers of hydrogen would have been expelled $\sim 20$ years ( $\sim 2$ years) before explosion given a CSM velocity of $\mathrm{v}_{\mathrm{csm}}=100(1000) \mathrm{km} \mathrm{s}^{-1}$. In the case of a longer rise time and $t_{\text {interact }}=150$ days, these values rise to $\sim 30$ years ( $\sim 3$ years). Overall we are looking for progenitors that would have lost their final hydrogen layers a matter of decades at most before the explosion. To keep our search broad, since many WR stars have wind velocities of several thousand $\mathrm{km} \mathrm{s}^{-1}$, we select all models which lost the last of their hydrogen 150 years before death.

\section{RESULTS}

After performing model selection according to the criteria described in the previous section, we find 2 excellent matches: a $30 \mathrm{M}_{\odot}$ primary with a $12 \mathrm{M}_{\odot}$ binary companion and a $30 \mathrm{M}_{\odot}$ secondary star with a $25 \mathrm{M}_{\odot}$ black-hole $(\mathrm{BH})$ - see Table 2 for a full summary of their properties. Both models follow a very similar evolutionary pathway; the main difference resides in the nature of the companion but qualitatively their life is identical and quantitatively they differ only marginally. The scenario with the main sequence companion is much more likely to occur from an IMF stand-point $-25 \mathrm{M}_{\odot}$ black holes are rarer than 12 solar mass MS stars. We can go further and quantify the odds of the primary model occurring over the secondary: one of the properties given for each BPASS stellar models is the number of stars that would be found in a $10^{6} \mathrm{M}_{\odot}$ population with the characteristics of that specific model. We find that the primary (secondary) progenitor model would apply to $1.58(0.014)$ stars per $10^{6} \mathrm{M}_{\odot}$, meaning that the odds of the first scenario occurring over the latter are $\sim 110: 1$. Consequently, in the rest of this section, we focus on the primary model.

Figure 1 shows the evolutionary track of the $30 \mathrm{M}_{\odot}$ star as well as its hydrogen content and radius evolution from the end of the main sequence onward. The system begins its life in a $\sim 5$-month orbit with a $428 \mathrm{R}_{\odot}$ separation. The main sequence is uneventful and the two stars essentially follow single star evolution, but after the onset of hydrogen shell burning, the radius of the primary increases rapidly up to $468 \mathrm{R}_{\odot}$ (see Figure 1 ). The transition to the red giant branch begins a common envelope phase that lasts 3800 years and strips the primary star of $12 \mathrm{M}_{\odot}$ of material. This mass loss episode changes the chemical make up of the progenitor - nearly $8 \mathrm{M}_{\odot}$ of hydrogen are lost to the interstellar medium and the hydrogen mass fraction falls from 0.74 to 0.43 . As a result of the mass loss, the radius of the progenitor stars decreases and the common envelope phase ends once it drops below $\sim 250$ solar radii. Roche lobe overflow and mass
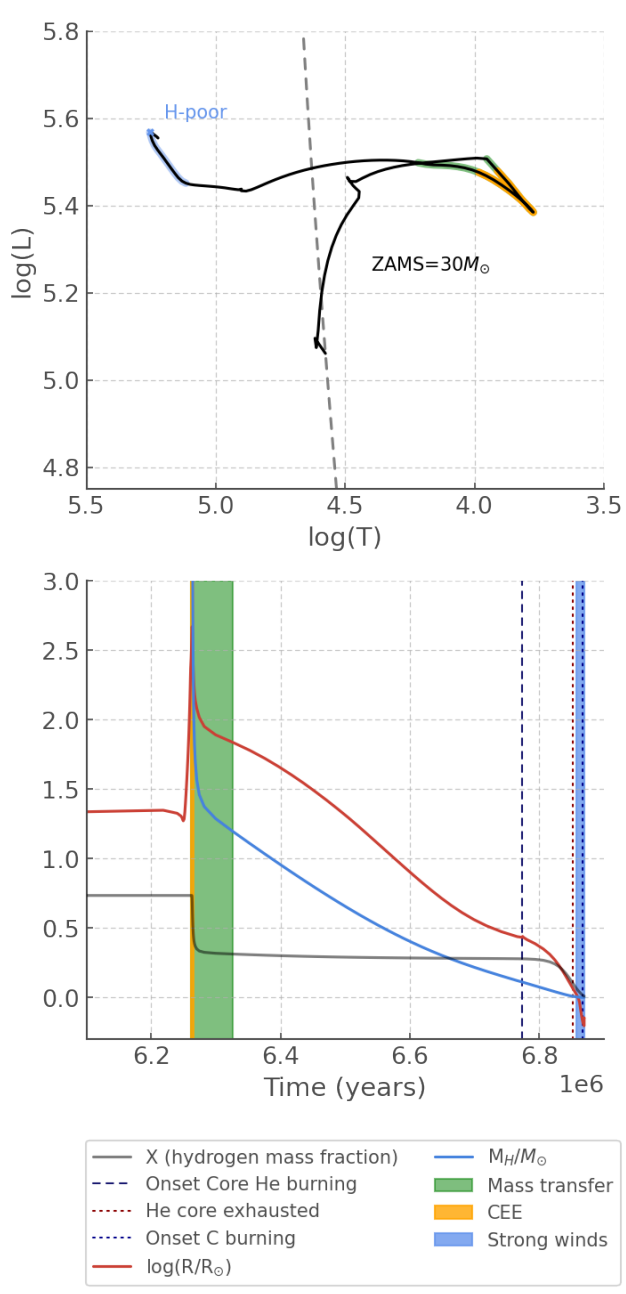

Figure 1. Top panel: Evolutionary tracks of the matching model. Bottom panel: evolution of the hydrogen content and radius from the end of the main sequence to the death of the star.

transfer continue for a further 60,000 years, causing an additional loss of $1.8 \mathrm{M}_{\odot}$ from the stellar envelope $\left(0.75 \mathrm{M}_{\odot}\right.$ of which hydrogen). The hydrogen mass fraction decreases marginally during this phase by $<0.1$.

When the system detaches its period is $\sim 50$ days and the progenitor has lost over half of its $\mathrm{M}_{\mathrm{ZAMS}}$, but the hydrogen content is still insufficiently low to explain a type I SN (or SLSN) explosion. Mass loss continues in steady winds with $\dot{M}=5.95( \pm 1.4) \times 10^{-6} \mathrm{M}_{\odot} / \mathrm{yr}$ for the next 0.5 Myrs, but a dramatic increase in luminosity in the last 6000 years raises $\dot{M}$ to $2 \times 10^{-5} \mathrm{M}_{\odot} / \mathrm{yr}$. This rise in luminosity begins after the exhaustion of the helium core and the onset of Helium shell burning, and it continues through carbon core burning (see Figure 1 ). It is this late rapid mass loss event that allows the hydrogen mass to drop below the threshold of $0.001 \mathrm{M}_{\odot}$, which is reached 54 years before explosion. With a kinetic energy of $10^{52}$ the ejecta mass reaches $10 \mathrm{M}_{\odot}$ which matches the ejecta mass of SN $2017 \mathrm{gci}$ very well despite our loose constraints.

The helium content at the end of our simulations amounts to $1.54 \mathrm{M}_{\odot}$ or 13.4 percent of the total mass of the model. It is worth noting that the BPASS models do not compute the full nucleosynthesis all the way to iron (in this model steps beyond the start of neon burning 3.5 months before explosion are not captured by the models) therefore the final helium content at explosion could differ slightly. 
Table 2. Properties of the two matching models.

\begin{tabular}{lll}
\hline Property & Primary progenitor & Secondary progenitor \\
\hline Primary $\mathrm{M}_{\text {ZAMS }}$ & $30 \mathrm{M}_{\odot}$ & $25 \mathrm{M}_{\odot} \mathrm{BH}$ \\
Secondary M MAMS & $12 \mathrm{M}_{\odot}$ & MS star $30 \mathrm{M}_{\odot}$ \\
$\mathrm{P}_{\text {ZAMS }}$ & 158 days & 63 days \\
$\mathrm{M}_{\mathrm{ej}}$ & $9.97 \mathrm{M}_{\odot}$ & $10.0 \mathrm{M}_{\odot}$ \\
$\mathrm{M}_{\text {rem }}$ & $1.45 \mathrm{M}_{\odot}$ & $1.46 \mathrm{M}_{\odot}$ \\
$\mathrm{X}$ & 0.0098 & 0.0099 \\
$\mathrm{M}_{\boldsymbol{H}}$ & $0.0004 \mathrm{M}_{\odot}$ & $0.0004 \mathrm{M}_{\odot}$ \\
$\mathrm{t}_{\text {csm }}$ & 54 years & 31 years \\
$\mathrm{P}_{\text {death }}$ & 64 days & 57 days \\
\hline
\end{tabular}

On the whole this echoes Yan et al. (2017) who remarked that if the hydrogen must be lost decades before the event, the progenitor will not have time to lose its entire helium envelope and it is likely that the ejecta of such SLSNe-I do contain helium. Large helium masses could remain invisible if there is not enough nickel to provide the non-thermal excitation required to excite helium lines (Lucy 1991) . In the context of type Ib/c SNe, Dessart et al. (2012) showed that even different levels of mixing could be enough to hide over $1 \mathrm{M}_{\odot}$ of helium in an ejecta $4-5 \mathrm{M}_{\odot}$. Therefore it is not unreasonable that $1.5 \mathrm{M}_{\odot}$ of helium would go unnoticed in a magnetar dominated SLSN with $10 \mathrm{M}_{\odot}$ of ejecta.

\section{DISCUSSION}

We used the explosion parameters derived by Fiore et al. (2021) for SN 2017gci to define search criteria to look for potential progenitors in the BPASS models (including both single and binary systems). The two matching stellar models we found were binary systems and if we perform the search on single-star-only models, no matches can be found. One of the crucial differences is in the nature of the remnant: where the binary models selected for NS remnants return 5851 (1861) matches for an explosion energy of $10^{52}\left(10^{51}\right)$ ergs, the single star models only provide 84 (3) matches. None of these are hydrogen free at the end of their lives. Tidal stripping in binary interactions is therefore an essential mass-loss step to yield the matching models. This is in line with the known importance of binary interactions for the production of other types of stripped supernovae (Dessart et al. 2012; Yoon 2015).

It is also interesting that the "normal" supernova $\left(10^{51} \mathrm{ergs}\right)$ prescription rapidly chokes the number of matching models (see Table 1). That is expected since a high kinetic energy input in the progenitor results in a lower remnant mass (making it easier to achieve the NS requirement) and a higher ejecta mass. Overall, this agrees with the kinetic energy of observed SLSNe (e.g. Nicholl et al. 2017) which is typically greater than $10^{51}$ ergs.

One of the main unknowns in our models is the precise description of the CSM resulting from the strong winds at the end of the life of the progenitor. Ideally the environment must be capable of producing the spectral lines and rebrightnening observed in SN 2017gci; CSM mass, opacity and geometry will all play a role. Modelling and deriving these parameters would be helpful in general, but could not aid in defining further search criteria in the BPASS models since the wind prescriptions used are not sufficiently detailed for this type of work. One of the main questions is that of the origins of the multiple brightening events observed in this type of SLSNe (e.g iPTF13ehe, SN2015bn, iPTF15esb, iPTF16bad - Yan et al. 2015; Nicholl et al. 2016; Yan et al. 2017). If they are indeed the result of shells of CSM, as has been previously suggested (Yan et al. 2015, 2017), then the stellar wind scenario needs to be able to produce shell-like geome-

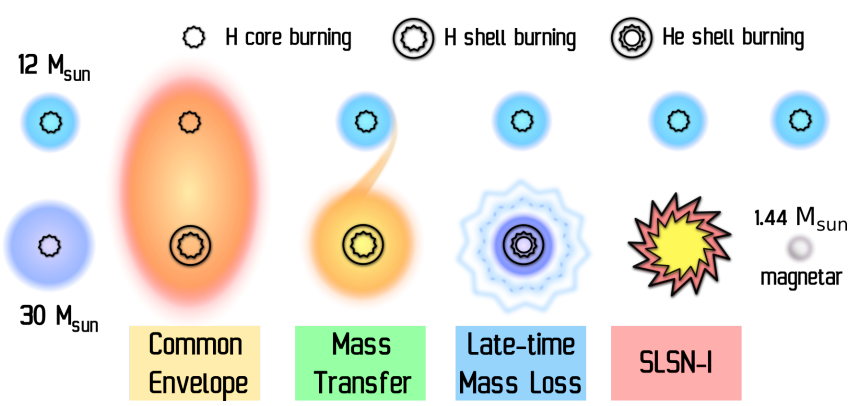

Figure 2. Evolutionary Pathway to the hydrogen poor superluminous SN $2017 \mathrm{gci}$

tries. Bow-shocks and photo-ionization confined shells could provide a natural explanation for this (Wilkin 1996; Mackey et al. 2014) but it would be strongly dependent on the environment and/or speed of the progenitor.

The potential presence of CSM around SLSN progenitors has previously been attributed to the pair-instability (PI) mechanism (for details on the mechanism see Woosley 2017). This is an attractive scenario as the CSM and bright explosion are both a consequence of the same phenomenon. However, the CSM and ejecta masses resulting from PI are much higher (e.g. Yan et al. 2015) than observed in the case of SN 2017gci and most PI explosions are nickel powered (which was excluded by Fiore et al. 2021) or CSM powered (which did not match the tail of the lightcurve). Our models can therefore fill areas of parameter space not covered by the PI models and these scenarios do remain relevant in a higher mass regime.

Finally, hydrogen poor SLSNe with bumpy tails are quite common and we therefore need any candidate pathway to be able to reproduce the numbers observed. Doing this in detail is not possible at this stage because, as mentioned above, it is likely that more than one pathway contributes to this type of transient, and it would require careful consideration of the star formation history associated with their host galaxy. That being said we can look at how rare the best matching model is using the model IMF calculated by BPASS: that is the number of systems one expects to find per million solar masses. For our primary model we find a model IMF of 1.58 , so for a host galaxy of mass between $10^{8}$ and $10^{9}$ (Perley et al. 2016), we expect between 150 and 1500 such systems if we assume a single burst of star formation. Consequently, the pathway described here is not rare by any means.

\section{CONCLUSION}

In this study we created search criteria (see Table 1) based on the explosion parameters of the hydrogen-poor superluminous SN $2017 \mathrm{gci}$ derived by Fiore et al. (2021) to find potential progenitors in the BPASS models. We found two $\mathrm{M}_{\mathrm{ZAMS}}=30 \mathrm{M}_{\odot}$ stellar models matching our constraints. Both are found in binary systems and single star models were not able to meet the constraints laid out in Section 3.

The most likely model (see Figure 2 ) has a $12 \mathrm{M}_{\odot}$ main sequence companion, whereas the other is a secondary star in orbit with a $25 \mathrm{M}_{\odot}$ black hole primary. We find that both progenitors have very similar pathways with a phase of common envelope evolution starting when the progenitor reaches a size of $\sim 250 \mathrm{R}_{\odot}$ followed by further mass transfer. Mass loss continues in winds and increases dramatically shortly before death as a result of a drastic increase in luminosity. 
These models are interesting for events such as SN 2017gci where a nickel powered explosion is unphysical and the ejecta mass relatively low $\left(\sim 10 \mathrm{M}_{\odot}\right)$. This renders scenarios such as pair-instability CSM previously invoked to explain the bumpy light-curve and late hydrogen features (Yan et al. 2015) inapplicable. It is also interesting to note that the progenitor here has a relatively low mass compared to other progenitor studies (e.g. Jerkstrand et al. 2017). The model in which hydrogen originates from a stripped companion described in (Moriya et al. 2015) used ejecta masses and energies very different from those found in SN 2017gci (see their table 1). A direct comparison is not appropriate as it was devised to match the specific observable of iPTF13ehe, so we cannot comment on the applicably or inapplicability of this scenario to SN $2017 \mathrm{gci}$ specifically, but it is worth noting that significant companion stripping from shock heating is expected for close companions (e.g. Hirai et al. 2014, 2018) and the Moriya et al. model could have larger applications.

A crucial difference between our progenitor search and that of other studies is that we do not explore an unconstrained parameter space to create a system that will best match a particular event; instead we searched existing simulations that simulate realistic stellar populations. Dedicated models can test specific aspects of the physics with more accuracy whilst wide scope simulations such as BPASS can check that certain pathways are consistent with stellar evolution at large. Both approaches are therefore essential if we are to fully uncover the progenitors of SLSNe.

\section{ACKNOWLEDGEMENTS}

The authors would like to offer warm thanks to the referee for their careful and insightful review. HFS would like to thank S.G. Parsons for interesting conversations and for their enduring support. HFS and JJE acknowledge the support of the Marsden Fund Council managed through Royal Society Te Aparangi. We are grateful to the developers of matplotlib, numpy (Hunter 2007; Harris et al. 2020) and pandas.

\section{DATA AVAILABILITY}

A Jupyter notebook containing our analysis is available ${ }^{3}$. The core data used for this work is contained in the following files in the data directory Stevance $(2020)^{4}:$ all_z_bin_bin_models, all_z_bin_sin_models

\section{REFERENCES}

Abbott B. P., et al., 2017, Phys. Rev. Lett., 119, 161101 Abbott B. P., et al., 2018, Phys. Rev. Lett., 121, 161101 Abbott B. P., et al., 2019, Phys. Rev. X, 9, 011001 Abbott R., et al., 2020, ApJ, 896, L44 Brennan S. J., et al., 2021, arXiv e-prints, p. arXiv:2102.09576 De Cia A., et al., 2018, ApJ, 860, 100

Denicoló G., Terlevich R., Terlevich E., 2002, MNRAS, 330, 69 Dessart L., Hillier D. J., Li C., Woosley S., 2012, MNRAS, 424, 2139

Eldridge J. J., Stanway E. R., 2016, MNRAS, 462, 3302

Eldridge J. J., Izzard R. G., Tout C. A., 2008, MNRAS, 384, 1109

\footnotetext{
3 https://github.com/UoA-Stars-And-Supernovae/Binary_ pathways_to_SLSNe_I_17gci

4 https://zenodo.org/record/4064300\#.YDyH5nVfgYt
}

Eldridge J. J., Stanway E. R., Xiao L., McClelland L. A. S., Taylor G., Ng M., Greis S. M. L., Bray J. C., 2017, Publ. Astron. Soc. Australia, 34, e058

Essick R., Landry P., Holz D. E., 2020, Phys. Rev. D, 101, 063007

Fiore A., et al., 2021, MNRAS, 502, 2120

Gal-Yam A., 2019, ARA\&A, 57, 305

Harris C. R., et al., 2020, Nature, 585, 357

Hirai R., Sawai H., Yamada S., 2014, ApJ, 792, 66

Hirai R., Podsiadlowski P., Yamada S., 2018, ApJ, 864, 119

Hunter J. D., 2007, Computing In Science \& Engineering, 9, 90

Inserra C., et al., 2013, ApJ, 770, 128

Inserra C., et al., 2017, MNRAS, 468, 4642

Jerkstrand A., et al., 2017, ApJ, 835, 13

Kasen D., Bildsten L., 2010, ApJ, 717, 245

Kroupa P., 2001, MNRAS, 322, 231

Lucy L. B., 1991, ApJ, 383, 308

Lunnan R., et al., 2018, Nature Astronomy, 2, 887

Ma X., Hopkins P. F., Kasen D., Quataert E., Faucher-Giguère C.-A., Kereš D., Murray N., Strom A., 2016, MNRAS, 459, 3614

Mackey J., Mohamed S., Gvaramadze V. V., Kotak R., Langer N., Meyer D. M. A., Moriya T. J., Neilson H. R., 2014, Nature, 512, 282

Maiolino R., et al., 2008, A\&A, 488, 463

Marino R. A., et al., 2013, A\&A, 559, A114

Moe M., Di Stefano R., 2017, ApJS, 230, 15

Moriya T. J., Liu Z.-W., Mackey J., Chen T.-W., Langer N., 2015, A\&A, 584, L5

Nicholl M., et al., 2015, MNRAS, 452, 3869

Nicholl M., et al., 2016, ApJ, 826, 39

Nicholl M., Guillochon J., Berger E., 2017, ApJ, 850, 55

Perley D. A., et al., 2016, ApJ, 830, 13

Pettini M., Pagel B. E. J., 2004, MNRAS, 348, L59

Stanway E. R., Eldridge J. J., 2018, MNRAS, 479, 75

Stanway E. R., Eldridge J. J., Becker G. D., 2016, MNRAS, 456, 485

Stevance H., 2020, BPASS Stellar Models in DataFrames, doi:10.5281/zenodo.4064300, https://doi .org/10.5281/zenodo. 4064300

Stevance H., Eldridge J., Stanway E., 2020a, The Journal of Open Source Software, 5, 1987

Stevance H. F., Eldridge J. J., McLeod A., Stanway E. R., Chrimes A. A., 2020b, MNRAS, 498, 1347

Tramper F., et al., 2015, A\&A, 581, A110

Wes McKinney 2010, in Stéfan van der Walt Jarrod Millman eds, Proceedings of the 9th Python in Science Conference. pp $56-61$, doi:10.25080/Majora-92bf1922-00a

Wilkin F. P., 1996, ApJ, 459, L31

Wofford A., et al., 2016, MNRAS, 457, 4296

Woosley S. E., 2010, ApJ, 719, L204

Woosley S. E., 2017, ApJ, 836, 244

Xiao L., Stanway E. R., Eldridge J. J., 2018, MNRAS, 477, 904

Xiao L., Galbany L., Eldridge J. J., Stanway E. R., 2019, MNRAS, 482, 384

Yan L., et al., 2015, ApJ, 814, 108

Yan L., et al., 2017, ApJ, 848, 6

Yoon S.-C., 2015, Publ. Astron. Soc. Australia, 32, e015

pandas development team T., 2020, pandas-dev/pandas: Pandas, doi:10.5281/zenodo.3509134, https://doi.org/10.5281/zenodo. 3509134

This paper has been typeset from a $\mathrm{T}_{\mathrm{E}} \mathrm{X} / \mathrm{L} \mathrm{T}_{\mathrm{E}} \mathrm{X}$ file prepared by the author. 